\title{
A Suggested Pathogenic Role for Trichosporon montevideense in a Case of Onychomycosis in a Japanese Monkey
}

\author{
Mohamed Mahdi ALSHAHNI ${ }^{1,2)}$, Koichi MAKIMURA ${ }^{1) *}$, Kazuo SATOH ${ }^{1)}$, Yumiko ISHIHARA ${ }^{1)}$, \\ Kosuke TAKATORI ${ }^{2}$, Tohru KIMURA ${ }^{3)}$ and Takuo SAWADA ${ }^{2)}$ \\ ${ }^{1)}$ Teikyo University Institute of Medical Mycology, 359 Otsuka, Hachioji, Tokyo 192-0395, ${ }^{2}$ Laboratory of Veterinary Microbiology, \\ Nippon Veterinary and Life Science University, Musashino, Tokyo 180-8602 and ${ }^{3)}$ Center for Experimental Animals, National Institutes \\ of Natural Sciences, 38 Nishigo-naka, Myodaiji, Okazaki, Aichi 444-8585, Japan
}

(Received 18 September 2008/Accepted 18 February 2009)

ABSTRACT. Onychomycosis is a fungal infection of fingernails or toenails caused by several species of fungi and yeasts. A Japanese monkey, Macaca fuscata, displayed severe onychomycosis in his 4 limbs. Diagnosis and etiological agent identification were performed by conventional and DNA-mediated methods. The accumulated findings of this case revealed Trichosporon montevideense, which has long been considered to be a nonpathogenic yeast. Here, we present the first report of an involvement of $T$. montevideense in an onychomycosis case.

KEY WORDS: Japanese monkey, Onychomycosis, Trichosporon montevideense.

Onychomycosis is a mycotic superficial invasion of keratinized tissue of the nail plate. Although it is commonly considered to be caused by the dermatophytic fungi, a variety of other organisms have been implicated as etiologic agents in the disease [7].

A 4-year-old male Japanese monkey, Macaca fuscata, showed severe onychomycosis in his four limbs. Clinically, some digits showed onychomycosis of the distal subungual type. However, most of the digits showed inflammation of both the nail and nail bed. In addition, some digits showed a purulent exudate and slight perilesional erythema (Fig. 1). This monkey had been used for neurological research, and it had not been possible to provide adequate veterinary care.

Trichosporon species are yeast-like organisms that belong to Basidiomycota. Several members of this genus are known to be pathogens $[8,10,15]$. The panel of diseases caused by Trichosporon spp. has been extended to include nail infection [15]. Several Trichosporon spp. have previously been reported to be causative agents of onychomycosis [9]. On the other hand, Trichosporon montevideense has long been considered to be a nonpathogenic yeast that naturally inhabits soil, water and human feces and skin [4]. Here, we describe for the first time an involvement of $T$. montevideense in a case of onychomycosis in a monkey.

Nail clippings were obtained and sent to our laboratory under aseptic conditions. A potassium hydroxide preparation $(\mathrm{KOH})$ was made by treating the nail scrapings with drops of $10 \% \mathrm{KOH}$-glycerol overnight at room temperature and then examined by optical microscopy.

Nail scrapings were cultured on Potato Dextrose Agar (PDA) supplemented with $50 \mu \mathrm{g} / \mathrm{m} l$ chloramphenicol and on Sabouraud Dextrose Agar (SDA) for up to 3 weeks at

\footnotetext{
* Correspondence to: Makimura, K., Teikyo University Institute of Medical Mycology, 359 Otsuka, Hachioji, Tokyo 192-0395, Japan.
}

e-mail: makimura@main.teikyo-u.ac.jp $28^{\circ} \mathrm{C}$. The cultures were macroscopically examined during the incubation period.

Total DNA was extracted from nail clippings by grinding them using a multibead shocker (MB524UAL; Yasui Kikai, Osaka, Japan) with $3 \mathrm{~g}$ of sterilized metal corn at $1800 \mathrm{rpm}$ for $20 \mathrm{sec}$. Ground nail clippings were soaked in lysis buffer (200 mM Tris-HCl, pH 8.0, 25 mM EDTA, 0.5\% w/v SDS and $250 \mathrm{mM} \mathrm{NaCl}_{2}$ ) and centrifuged at $10,000 \times \mathrm{g}$ for $5 \mathrm{~min}$. The supernatant was extracted with phenol:chloroform:isoamyl alcohol $(25: 24: 1)$ and subsequently extracted with chloroform. Finally, DNA was precipitated with an equal volume of 2-propanol at $-20^{\circ} \mathrm{C}$ for $10 \mathrm{~min}$, washed with $150 \mu l$ of $70 \%$ ethanol, dried and suspended in Ultrapure water.

The grown colonies and the total DNA extracted from nail were subjected to DNA-mediated identification target-

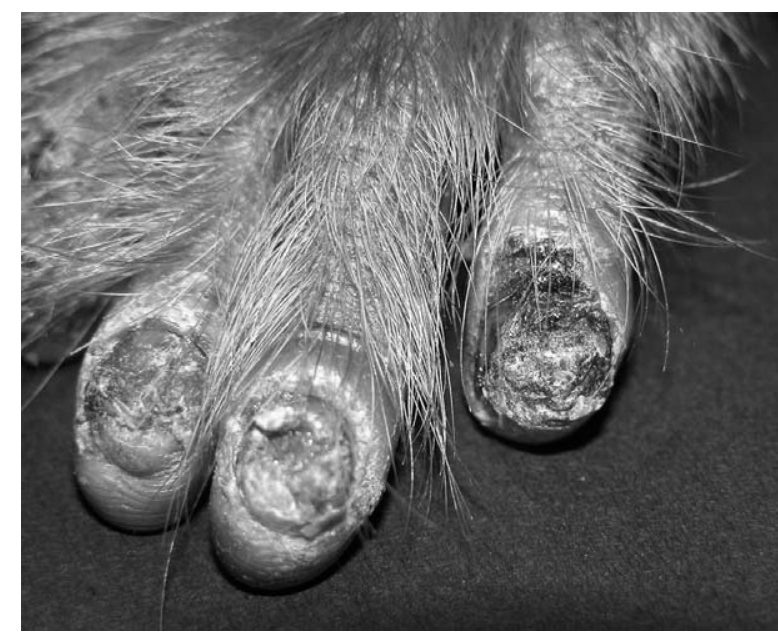

Fig. 1. Onychomycosis in the fingernails of the diseased Japanese monkey. 
ing the $28 \mathrm{~S}$ region of ribosomal RNA gene (rDNA) with a pair of primers, 28SF1 (5'-AAGCATATCAATAAGCGGAGG-3') and 635 (5'-GGTCCGTGTTTCAAGACGG-3') [13]. Colony number 5, grown on SDA, was identified by a set of bacterial universal primers, F3L (5'-GTCCCGCAACGAGCGCAAC-3') and 1492R (5'-CGGTTACCTTGTTACGACTT-3') [6, 12] since we could not generate PCR products by the above-described fungal primers. Sequence similarity was searched using the Basic Local Alignment Search Tool (BLAST) (http://blast.ncbi.nlm.nih.gov/ Blast.cgi) to identify the generated sequences [1].

Physiological and morpholgical identification of colony number 2 were performed [5]. Its ability to grow on a sole carbon source was checked by an auxanographic method in which $20 \times 10^{4} / \mathrm{m} l$ of freshly prepared yeast suspended in yeast nitrogen base (DIFCO, U.S.A.) and incorporated with agar cooled to $45^{\circ} \mathrm{C}$. A quantity of $5 \mathrm{mg}$ of 30 carbon sources was spotted on the surface of the previous yeast

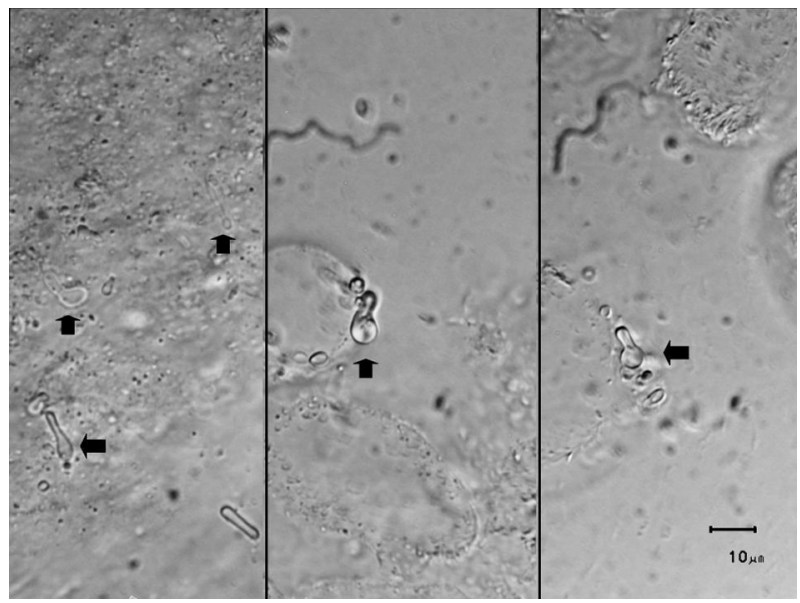

Fig.2. Direct microscopic examination of nail clippings prepared using $\mathrm{KOH}$-glycerol overnight at room temperature. Different fungal conidia are indicated by arrows. incorporated medium. Cultures were incubated at $25^{\circ} \mathrm{C}$, and results were read after 2-4 days. Moreover, this isolate was cultivated on SDA medium under different incubation temperatures, $28^{\circ} \mathrm{C}, 35^{\circ} \mathrm{C}$ and $37^{\circ} \mathrm{C}$. A slide culture was prepared on $2 \%$ malt extract agar and incubated for 2 days at $28^{\circ} \mathrm{C}$.

Direct microscopic examination of nail clippings uncovered pyriform to spherical elongated conidia (Fig. 2). Budding cells were also present. Macroscopic examination after 5-7 days of incubation revealed three types of colonies on PDA, 1) pink smooth moist mucoid colonies, 2) cream-colored moist soft colonies that changed into colonies with a deeply fissured marginal zone after a long period of incubation and 3) white-dry colonies, while it revealed two types of colonies on SDA, 4) creamy-colored and 5) white-dry colonies. Molecular analysis of these colonies and BLAST sequence similarity search identified the above-described colonies as 1) Rhodotorula mucilaginosa, 2) Trichosporon montevideense, 3) Torulaspora delbrueckii, 4) Trichosporon montevideense and 5) Leuconostoc mesenteroides, respectively (Table 1). On the other hand, the total DNA from the nails resulted in two fungal species, T. montevideense and Trichosporon caseorum, based on the $28 \mathrm{~S}$ region (Table 1).

The ability of our T. montevideense isolate to assimilate variant carbon sources is illustrated in Table 2. Furthermore, it was able to grow at $28^{\circ} \mathrm{C}$ and $35^{\circ} \mathrm{C}$ but not at $37^{\circ} \mathrm{C}$. Microscopically, the slide culture examination showed hyphae disarticulated into arthroconidia and budding cells (Fig. 3).

The conventional method for diagnosis of an onychomycosis has to be performed by demonstrating the presence of causative agents in clinical materials using microscopic examination, which helps to rule out the non-fungal causes of nail disease, and by culture mediated identification to determine the exact pathogen that is responsible for the onychomycosis case [3]. In the current case, direct microscopy of clinical materials provided evidence of fungal invasion of

Table 1. The accession numbers and maximum identities of the species identified from nail clippings of the diseased monkey

\begin{tabular}{lclcl}
\hline No. & Source & Accession No. ${ }^{\text {h) }}$ & Maximum identity & Species name \\
\hline $1^{\text {a) }}$ & PDA & EU807914.1 & $100 \%$ & Rhodotorula mucilaginosa \\
\hline $2^{\text {b) }}$ & PDA & AF105397.1 & $100 \%$ & Trichosporon montevideense \\
\hline $3^{\text {c) }}$ & PDA & EF063125.1 & $100 \%$ & Torulaspora delbrueckii \\
\hline $4^{\text {d) }}$ & SDA & AF105397.1 & $100 \%$ & Trichosporon montevideense \\
\hline $5^{\text {e) }}$ & SDA & EU074837.1 & $98 \%$ & Leuconostoc mesenteroides \\
\hline $6^{\text {f) }}$ & Nail $^{\text {i) }}$ & AF105397.1 & $100 \%$ & Trichosporon montevideense \\
\hline $7^{\text {g) }}$ & Nail $^{\text {i) }}$ & AJ319757.1 & $99 \%$ & Trichosporon caseorum \\
\hline
\end{tabular}

a) Pink smooth moist mucoid colonies, b) cream-colored moist soft colonies and c) white-dry colonies were all isolated on PDA. d) Creamy-colored colonies and e) white-dry colonies were isolated from SDA. f)-g) Fungal species identified directly from nail clippings by DNA extraction. h) The accession numbers of the identified fungal species from colonies and from the DNA of nail clippings based on BLAST sequence similarity search. i) Refers to the total DNA extracted directly from the nails. 
Table 2. Results of carbon assimilation tests for identification of our T. montevideense isolate

\begin{tabular}{lclc}
\hline Tested substances & Results & Tested substances & Results \\
\hline Glucose & + & D-Arabinose & - \\
\hline Galactose & + & D-Ribose & + \\
\hline L-Sorbose & - & L-Rhamnose & - \\
\hline Sucrose & + & N'^-Acetyl-D-glucosamine & + \\
\hline Maltose & + & Methanol & - \\
\hline Cellobiose & + & Ethanol & - \\
\hline Trehalose & - & Glycerol & - \\
\hline Lactose & + & Erythritol & - \\
\hline Melibiose & - & Ribitol & - \\
\hline Raffinose & - & Galactitol & + \\
\hline Melezitose & + & D-Mannitol & + \\
\hline Inulin & - & D-Glucitol & + \\
\hline Soluble starch & + & a-Methyl-D-glucoside & - \\
\hline D-Xylose & + & Salicin & - \\
\hline L-Arabinose & + & Inositol & + \\
\hline & & DL-Lactate & -
\end{tabular}

Substances in bold are the identical materials for T. montevideense idenfication. Plus (+) and minus (-) correspond to ability (or disability) of the isolated T. montevideense to use carbon sources.

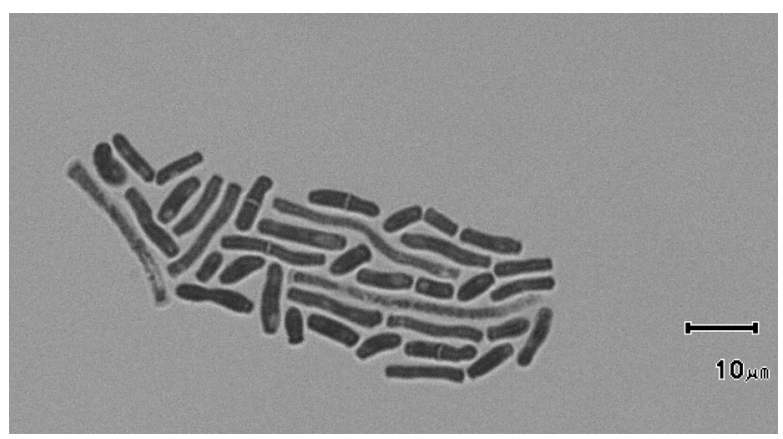

Fig. 3. Slide culture of Trichosporon montevideense isolated from nail clippings on PDA. The slide shows hyphae disarticulating into arthroconidia. This slide was prepared on $2 \%$ malt extract agar after 2 days of incubation at $28^{\circ} \mathrm{C}$.

the tissue. However, culture of nail clippings resulted in more than one type of colony. Identifying the resulting colonies by molecular biology was performed using fungal universal primers and BLAST sequence similarity search. Bacterial universal primers had to be employed for colonies that did not respond to the previous primers. Simultaneously, we tried to identify causative agents directly from nails to exclude agents that cultured on media as contaminants, but were not involved in the infection, by direct analysis of DNA extracted from the nails, which revealed two types of fungal species (Table 1).
Although many fungal organisms were identified in culture and/or directly based on the DNA extracted from the nail clippings, T. montevideense is the only agent suspected to be responsible for the case of onychomycosis described here. Demonstration of the presence of fungal elements directly in the nails excludes $L$. mesenteroides form being the pathogen in this case. In addition, isolation of $R$. mucilaginosa on PDA was coincidental, as it could not be isolated repeatedly on SDA, and it was not identified in the DNA extracted from the nail clippings. On the other hand, Rhodotorula spp. are well-known contaminants of clinical isolates, and have no history of superficial infections $[2,15]$. $T$. delbrueckii is a nonpathogenic yeast that is mainly involved in the fermentation processes and wine production, and it could not be identified directly in DNA isolated from the nails or on SDA media [11]. T. caseorum has been reported in a few studies as a new species isolated from cheese, but since it could not be isolated on non-selective media (SDA and PDA), the possibility of it having a pathogenic role in this case is poor.

In contrast, $T$. montevideense has previously been described to be associated with summer-type hypersensitivity pneumonitis but not as a real pathogen [14]. The morphologies of the yeasts seen in direct microscopic examination of nail clippings and the yeasts displayed in slide culture of T. montevideense, which was isolated from the nails, were nearly symmetrical (Figs. 2, 3). Nevertheless, $T$. montevideense was the only species isolated from all cultures and isolated directly from the DNA of nail clippings, and it had the same accession number in all isolations (Table 1).

The identification of the $T$. montevideense strain was proved not only on molecular biologcial grounds but also on physiolcogical and morphologcial bases. Homological search by BLAST of the isolated T. montevideense resulted in $100 \%$ maximum identity based on the $28 \mathrm{~S}$ of the rDNA region. In addition, it assimilated galactose, while it could not grow in the presence of L-sorbose, L-rhamnose, melibiose and erythritol, which are identifying to T. montevideense [4]. Conversely, it could not assimilate ethanol, glycerol, ribitol, a-Methyl-D-glucoside, salicin and inositol, and this might be attributed to a unique phenotype of this strain. These findings, in addition to the typical microscopic features of $T$. montevideense and the ability to grow at $35^{\circ} \mathrm{C}$ but not at $37^{\circ} \mathrm{C}$, confirm the idenfication of our strain.

Trichosporonosis is disease caused by Trichosporon species. The infection manifests as disseminated or superficial mycoses (white piedra) [2, 15]. Several species of this genus are known to be pathogens of humans and animals [8, $9,15,16]$. Recently, this genus has undergone taxonomic revision through which the number of species considered as pathogens has been increased from one to seven, which are T. cutaneum, T. asahii, T. mucoides, T. inkin, T. ovoides, T. asteroids and $T$. loubieri $[2,8,10,15]$. This leaves the door open to reconsider the pathogenicity of the other members of this genus in light of new reports. Considering all our findings, T. montevideense has a higher possibility of having 
a pathogenic role in this case.

In conclusion, Trichosporon montevideense has long been considered to be a nonpathogenic yeast. However, the isolation of T. montevideense in this case of onychomycosis in a monkey indicated that this isolate was possibly a pathogenic organism. To our knowledge, this is the first report to implicate of T. montevideense in an onychomycosis case.

\section{REFERENCE}

1. Altschul, S.F., Gish, W., Miller, W., Myers, E.W. and Lipman, D.J. 1990. Basic local alignment search tool. J. Mol. Biol. 215: 403-410.

2. Davise, H. L. 2002. Detailed description-yeast and yeastlike organisms. pp.109-143. In: Medically Important Fungi, a Guide to Identification, 4th ed. ASM Press, Washington, D.C.

3. Elewski, B. 1998. Onychomycosis: Pathogenesis, Diagnosis, and Management.Clin. Microbiol. Rev. 11: 415-429.

4. Gueho, E., Smith, M.Th. and De Hoog, G. S. 1998. Trichosporon Behrend. pp 854-871. In: The Yeasts: Taxonomic Study, 4th ed. ( Kurtzman, C.P. and Fell, J.W. eds), Elsevier Science, The Netherlands.

5. Yarrow, D. 1998. Methods for the isolation, maintenance and identification of yeasts. pp 77-100. In: The Yeasts: Taxonomic Study, 4th ed. ( Kurtzman, C.P. and Fell, J.W. eds), Elsevier Science, The Netherlands.

6. Gürtler, V. and Stanisich, V. A. 1996. New approaches to typing and identification of bacteria using the $16 \mathrm{~S}-23 \mathrm{~S}$ rDNA spacer region. Microbiology 142: 3-16.

7. Jennings, B. M., Weinberg, M. J., Koestenblatt, K. E. and Lesczczynski, C. 2002. Study of Clinically Suspected Onychomycosis in a Podiatric Population. J. Am. Podiatr. Med. Assoc. 92: $327-330$.

8. Kevin, C. H. and Howell, S. A. 2002. Yeasts: Blastomycetes and Endomycetes. pp. 499-533. In: Pathogenic Fungi in Humans and Animals, 2nd ed. (Howard, D. H. ed.), Marcel Dekker, Inc. New York, Basel.

9. Kim, E. S., Kim, D. H., Chang, S. E., Lee, M. W., Choi J. H., Sung, K. J., Moon, K. C. and Koh, J. K. 2003. Trichosporon Species in Onychomycosis and Tinea Pedis. Korean J. Dermatol. 41: 702-707.

10. Marty, F. M., Barouch, H. D., Coakley, P. E. and Baden, R. L. 2003. Disseminated trichosporonosis caused by Trichosporon loubieri. J. Clin. Microbiol. 141: 5317-5320.

11. Ohshima, Y., Sugaura, T., Horita, M., Sasaki, T. 1987. Industrial Application of Artificially Induced Diploid Strains of Torulaspora delbrueckii. Appl. Environ. Microbiol. 53: 1512 1514.

12. Sasoh, M., Masai, E., Ishibashi, S., Hara, H., Kamimura, N., Miyauchi, K. and Fukuda, M. 2006. Characterization of the terephthalate degradation genes of Comamonas sp. Strain E6. Appl. Environ. Microbiol. 72: 1825-1832.

13. Sugita, C., Makimura, K., Murakami, A., Murai, Y., Yamaguchi, H. and Nagai, A. 2003. A case of pulmonary aspergilloma molecular biological identification and typing of the isolates from antemortem sputa and autopsy fungus ball. Mycoses 46: 149-152.

14. Sugita, T., Ikeda, R. and Nishikawa, A. 2004. Analysis of Trichosporon isolates obtained from the houses of patients with summer-type hypersensitivity pneumonitis. J. Clin. Microbiol. 42: $5467-5471$.

15. Vazquez, A. J. 2003. Rhodotorula, Malassezia, Trichosporon, and Other Yeast-Like Fungi. pp. 206-217. In: Clinical Mycology (Dismukes, W. E., Pappas, P. G. and Sobel, J. D. eds.), Oxford University Press, Inc. New York, New York 10016.

16. Vijaya, D., Kumar Anand B. H., Nagarathamma, T. and Joseph, M. 2000. Onychomycosis caused by Trichosporon beigelii. Ind. Assoc. Dermatol. Venereol. Leprol. 66: 93-94. 